Supporting Information for

\title{
Artificial Graphene on Si Substrates: Fabrication and Transport
}

\section{Characteristics}

Peizong Chen, Ningning Zhang, Kun Peng, Lijian Zhang, Jia Yan, Zuimin Jiang and Zhenyang Zhong*

The 2D QD array of AG simplified to be a pseudo-1D ordered system

For bias voltages $\mathrm{V}$ above a threshold voltage $\mathrm{V}_{\mathrm{T}}$, the $\mathrm{I}-\mathrm{V}$ curve of a QD or nanoparticle array can be fitted by a power law, ${ }^{1-5}$

$$
I=C\left(V / V_{\mathrm{T}}-1\right)^{\xi}
$$

where $C, V_{T}$ and $\xi$ are fitting parameters. This I-V characteristics originates from the accessible current-conducting paths via series-parallel combination of many tunneling among QDs throughout the array. In addition, the index $\xi$ reflects the transverse correlation of the current paths, which is associated with the coupling strength and the network topology, as well as the disorders in QD arrays. ${ }^{1-5}$ An analytical model predicts the index $\xi=1$ and 5/3 for 1D and 2D QD arrays in a square lattice, respectively. ${ }^{2}$ For 2D QD arrays, previous experiments exhibit sample-dependent index $\xi$ in a wide range from 1.4 to $2.2 .^{1,3}$ The main reason is the different disorders in QD arrays, which affect the coupling among QDs and the network topology in the QD array. In general, 
disorders lead to the increase of current paths with reduced lateral region. The smaller index $\xi$ indicates the smaller number of current paths with the larger lateral regions, which demonstrates the less disorders and the strong coupling in 2D QD arrays. The limitation is $\xi=1$ for ideally ordered QD arrays, meaning that all QDs in the 2D array are involved in a single current path and the 2D QD array acts as a 1D array. ${ }^{3}$ In our cases, the index $\xi$ of $\sim 1.1$ is extracted by fitting the experimental data, as demonstrated in the main text. It is much smaller than previous ones. ${ }^{1-5}$ Moreover, it is close to 1 and temperature-independent. Accordingly, under the bias voltage, the 2D QD array of present AG can be regarded as a pseudo-1D system due to the strong coupling and/or the network topology of honeycomb geometry in the AG with much less disorders than previous cases. To extract the quantitative relationship between the index $\xi$ and the amount of disorders in the 2D QD arrays, further studies are highly desired. For sufficient holes in QDs, their rearrangement under an external electric field can result in the nearly same relative quantum energy shift between the nearest neighboring QDs in the AG at bias voltages. Accordingly, the QDs in a 2D honeycomb lattice can be simplified to be a $1 \mathrm{D}$ ordered system with a uniform electric field.

\section{References:}

1. Parthasarathy, R.; Lin, X.-M.; Jaeger, H. M. Electronic Transport in Metal Nanocrystal Arrays: The Effect of Structural Disorder on Scaling Behavior. Phys. Rev. Lett. 2001, 87,186807. 
2. Middleton, A. A.; Wingreen, N. S. Collective Transport in Arrays of Small Metallic Dots. Phys. Rev. Lett. 1993, 71, 3198-3201.

3. Black; C., T. Spin-Dependent Tunneling in Self-Assembled Cobalt-Nanocrystal Superlattices. Science 2000, 290, 1131-1134.

4. Fan, H.; Yang, K.; Boye, D. M.; Sigmon, T. W.; Malloy, K. J.; Xu, H.; Lopez, G. P.; Brinker, C. J. Self-Assembly of Ordered, Robust, Three-Dimensional Gold Nanocrystal/Silica Arrays. Science 2004, 304, 567-571.

5. Staley, N. E.; Ray, N.; Kastner, M. A.; Hanson, M. P.; Gossard, A. C. ElectricField-Driven Insulating-to-Conducting Transition in a Mesoscopic Quantum Dot Lattice. Phys. Rev. B 2014, 90 (19),195443. 